\title{
The prevalence of thermotolerant Campylobacter species in food animals in Jimma Zone, southwest Ethiopia
}

\author{
Tesfaye Kassa ${ }^{1}$, Solomon Gebre-selassie ${ }^{1}$, Daniel Asrat ${ }^{2}$
}

\begin{abstract}
Background: Thermotolerant Campylobacter spp. is known to occur in the intestinal systems of a wide variety of domestic and wild animals. Although Campylobacter jejuni and Campylobacter coli cause acute diarrhoeal diseases in humans worldwide, they mostly manifest themselves in an apparently healthy carrier state in other mammalian species. However, little is known about the presence of campylobacter bacteria in various food animals as possible sources of infection to humans in Ethiopia.

Objective: The aim of this study is to determine the prevalence of thermotolerant Campylobacter spp. in various food animals in Jimma zone, southwest Ethiopia.

Methods: A cross-sectional study was conducted in urban and rural farm animal settings in Jimma, southwest Ethiopia in the period between January 2004 to April 2004. Fecal specimens were collected from 485 various food animals (cattle, $\mathrm{n}=205$; poultry, $\mathrm{n}=191$; pigs, $\mathrm{n}=18$; sheep $\mathrm{n}=71$ ) and cultured using standard methods.

Results: Campylobacter spp. were isolated from 192 (39.6\%) out of 485 fecal specimens taken from various urban and rural farm animals. The highest isolation rate was recorded among chickens $(68.1 \%)$, followed by pigs $(50.0 \%)$, sheep (38.0\%) and cattle (12.7\%). Among the 192 thermophilic campylobacters isolated, 135 (70.3\%) were identified to be C. jejuni, 51 (26.6\%) were C. coli and $6(3.1 \%)$ were C. lari. C. jejuni was the most prevalent species in chickens $(80.8 \%)$, followed by sheep $(59.3 \%)$ and cattle $(53.8 \%)$. All isolates found in pigs were identified to be $C$. coli $(100 \%)$.

Conclusion: The results of this study indicate that thermophilic campylobacters are very frequent among various food animals in Ethiopia, suggesting possible risks of infection to people through the consumption of contaminated animal products or through contact with infected animals. [Ethiop.J.Health Dev. 2005;19(3):225-229]

\section{Introduction}

The thermophilic campylobacters- C. jejuni and C. coli account for the majority of gastrointestinal diseases attributed to the genus Campylobacter. Campylobacter is now considered to be one of the causes of diarrhoeal diseases in humans, resulting mainly from the contamination of poultry and other animal products (1, 2). However, the pathogenesis of Campylobacter gastroenteritis is not fully characterized (3). A serious consequence of diarrhoeal diseases is the Guillain-Barrè syndrome (GBS) characterized by polyneuritis of the peripheral nerves, which may lead to either short-term or lengthy paralysis (4). Campylobacter received serious attention as a causative agent of diarrhoea only since 1973 with the introduction of a selective medium for isolation from human stool (5). A total of 15 species and six subspecies are now categorized in the genus Campylobacter and 12 of these species are associated with human diseases $(6,7)$. Only the five significant human pathogens are considered in detail,-the enteropathogens C. jejuni, C. coli, C. lari, C. upsalinesis, and the systemic pathogen C. fetus (3). Campylobacters are small gram-negative, non-spore-forming, helical bacteria with a distinctive 'darting' motility, and are catalase and oxidase positive. Campylobacter spp. can be

oral cavity of animals and humans $(7,8)$. These organisms are widely distributed in nature and are mainly recognized as zoonotic infections in a multitude of animal reservoirs, particularly avian species, with shedding into the environment. Although $C$. jejuni and $C$. coli cause acute diarrhoeal diseases in people worldwide (9), they are mostly present in an apparently healthy carrier state in other mammalian species (10). Humans acting as accidental hosts typically acquire these potential pathogens through the ingestion of contaminated food, particularly poultry, unpasteurized milk, improperly treated water, or animal contact with sporadic infections far outnumbering those occurring in point source epidemics $(11,12)$. The few reported studies of Campylobacter spp. as human enteric pathogens in Ethiopia showed isolation rates ranging from $13.6 \%$ to $13.8 \%(13,14)$. Several countries have reported the epidemiology of Campylobacter bacteria in different wild and domestic animals $(10,15-17)$. However, little is known about the presence of Campylobacter spp. in various food animals as possible sources of infection for humans in Ethiopia. Therefore, the present study was undertaken to determine the prevalence of thermotolerant Campylobacter species in cattle, sheep, pigs and chickens in Jimma zone, southwest Ethiopia.
\end{abstract} found in the reproductive organs, intestinal tracts, and

\footnotetext{
${ }^{1}$ Department of Medical Microbiology, Immunology and Parasitology, Faculty of Medical Sciences Jimma University, P. O. Box. 378, Jimma, Ethiopia; ${ }^{2}$ Department of Medical Microbiology, Immunology and Parasitology, Faculty of Medicine, Addis Ababa University, P.O. Box. 9086, E-mail asratdaniel@telecom.net.et, Addis Ababa, Ethiopia
} 


\section{Methods \\ Study area}

A cross-sectional study was conducted in urban and rural farm animal settings in Jimma, southwest Ethiopia during the period from January 2004 to April 2004. The urban farm animal settings were located at Jimma University College of Agriculture (JUCA) and the Jimma Dairy Development Enterprise (JDDE), while the rural animal farm settings were located at the Merrewa Peasant Association located about $10 \mathrm{~km}$ north of Jimma town.

\section{Collection of samples}

As outlined in Table 1, a total of 485 fresh faecal samples/rectal swabs were collected from apparently healthy rural and urban farm animals. The 112 cattle, 94 chickens and 71 sheep from the Merewa rural setting $(n=277)$ and the 93 cattle, 97 chickens and 18 pigs from the urban setting $(\mathrm{n}=208)$ were included in the study. Approximately 1-5 grams of fecal samples were obtained using direct rectal retrieval and rectal swab systems. The samples were taken with a sterile cotton wool swab moistened in nutrient broth and transported in CarryBlair Transport medium (Oxoid Ltd, Basingstoke, Hampshire, England). The sample size was calculated based on the reported mean carrier rate in different food animals to be $50 \%$ or higher (18).

\section{Culture and identification of thermotolerant Campylobacter spp}

All fecal samples/ rectal swabs obtained from different food animals were inoculated directly onto Modified Charcoal Cefoperazone Deoxycholate Agar (Blood free selective medium) (Oxoid Ltd, Basingstoke, Hampshire, England), which is appropriate for the isolation of thermophilic campylobacters (19). The cultures were incubated in a microaerophilic atmosphere, achieved in anaerobic jars (Oxoid, UK) without catalyst and by using CampyGen ${ }^{\circledR}$ gas generating kits $\left(5 \% \mathrm{O}_{2}\right.$ and $\left.10 \% \mathrm{CO}_{2}\right)$ (Oxoid, UK) at $42^{\circ} \mathrm{C}$ for 48 hours. The growth of thermophilic campylobacters was detected by their characteristic appearance on culture media i.e. the presence of flat grayish colonies resembling droplets of water sprayed on the medium. Preliminary identification was performed based on the characteristic Gram-staining reactions and positive tests for oxidase and catalase agents. The type strains $C$. jejuni (NCTC 11351), C. coli (LMG 6440) and C. lari (NCTC 11352) were included as positive controls.

All the isolated campylobacter strains were kept frozen at $-70^{\circ} \mathrm{C}$ as stab culture in $1 \%$ nutrient gar (Oxoid, UK) until species differentiation was done.

\section{Species differentiation}

All isolated strains identified as thermophilic campylobacters were tested for hippurate hydrolysis, $\mathrm{H}_{2} \mathrm{~S}$ production and susceptibility to nalidixic acid and cephalothin. These parameters formed the basis for the identification of C. jejuni, C. coli or C. lari, as proposed by On et al. (7). For the hippurate hydrolysis test, a 48hour pure culture taken from blood agar plates was duly emulsified in $1 \%$ sodium hippurate and incubated in a heating block at $37^{\circ} \mathrm{C}$. After 2 hours of incubation, $0.2 \mathrm{ml}$ of the ninhydrin reagent was slowly added on the sides of the tubes. Without mixing, the tubes were returned to the heating block for 20 minutes and examined immediately, without shaking for color development. A deep purple crystal violet like color, which indicates the presence of glycine that results from the hydrolysis of hippurate, was considered a positive test. Those organisms yielding a positive test were considered $C$. jejuni, while those organisms that showed a negative reaction (no color development) were considered C. coli and C. lari.

For hydrogen sulfide $\left(\mathrm{H}_{2} \mathrm{~S}\right)$ production test, pure culture was inoculated on a triple sugar iron agar (Oxoid, UK) and incubated at $37^{\circ} \mathrm{C}$. Hydrogen sulfide production turned parts of the agar black indicating a positive test. All the tested campylobacter strains were found to be negative for $\mathrm{H}_{2} \mathrm{~S}$ production.

Susceptibility tests to nalidixic acid $(30 \mu \mathrm{g})$ (Oxoid, UK) and cephalothin $(30 \mu \mathrm{g})(\mathrm{Oxoid} \mathrm{UK})$ were performed for all isolates of Campylobacter spp. in accordance with the criteria set by the National Committee for Clinical Laboratory Standards (NCCLs) using the disk diffusion method (20). The isolates were classified as sensitive and/or resistant according to the standardized tables supplied by the NCCLs (20). Campylobacter strains that were sensitive to nalidxic acid but which are resistant to cephalothin were considered C. jejuni and C. coli, while strains that were resistant to both drugs were considered C. lari.

\section{Statistical analysis}

The Epi info version 2000 (approved by CDC, Atlanta, Georgia, USA) was used for statistical analysis. A ChiSquare test was applied to examine whether the differences between the values were significant.

\section{Results}

\section{Isolation of Campylobacter spp}

The numbers and percentages of the theromophilic campylobacters isolated from 485 different food animals are presented in Table 1. Campylobacter spp. were isolated from $192(39.6 \%)$ of the 485 fecal samples investigated from various urban and rural farm animals. The highest isolation rate was recorded among chickens (68.1\%), followed by pigs $(50.0 \%)$, sheep $(38.0 \%)$ and cattle (12.7\%). The rate of Campylobacter spp. isolated in all animals was found to be higher in animals from urban areas than from rural settings $(56.7 \%$ vs. $26.7 \%$, $\mathrm{p}<0.05)$.

\section{Phenotype distribution}

Among the 192 thermophilic Campylobacter strains 
isolated from various food animals, 135 (70.3\%) were found to be $C$. jejuni, $51(26.6 \%)$ were $C$. coli and 6 (3.1\%) were found to be C. lari (Table 2). The number of C. jejuni, C. coli and C. lari isolated per food animal species were: cattle, 14/10/2; chickens, 105/21/4; pigs, $0 / 9 / 0$ and sheep, 16/11/0. respectively. C. jejuni was found to be the most prevalent species in chickens $(80.8 \%)$, followed by sheep $(59.3 \%)$ and cattle $(53.8 \%)$. All isolates found in pigs were C. coli $(100 \%)$.

\section{Discussion}

Many different animal species maintain Campylobacter spp. without showing clinical signs (18). The most important species of Campylobacter in veterinary medicine are C. fetus subsp. fetus and venerealis (21). Of the Campylobacter spp. that are pathogenic in food animals, $C$. fetus could cause reproductive disorders such as abortion and infertility in cattle and sheep (21), and $C$. hyointestinalis and C. mucosalis have been associated with enteritis in pigs and cattle (21). But, the role of $C$. jejuni as a primary pathogen in farm animals is uncertain (18). C. jejuni could be found in the faeces of diarrheic and healthy calves and piglets, but both $C$. jejuni and $C$. coli can cause a mild self-limiting enteritis and bacteremia when inoculated orally in to new born calves (18). Isolation of C. jejuni, C. coli and C. lari from the intestinal contents of domestic livestock revealed considerable intra-and interspecies variation (10).

Overall, this study has found a high frequency of Campylobacter spp. in chickens (68.1\%) (Table 1). The reported prevalence of Campylobacter bacteria in broiler flocks ranged between $35-57 \%$ in Europe (22) and 64$100 \%$ in Africa $(17,23,24)$. In this study, among the thermophilic campylobacters isolated from chickens, $C$. jejuni accounted for $80.8 \%$, C. coli for $16.2 \%$ and C. lari for $3.0 \%$ (Table 2). Similar results have also been reported in other countries indicating a higher prevalence of $C$. jejuni than C. coli in poultry farms (18). Poultry appeared to be significant sources of Campylobacter bacteria, and chickens were found to be healthy carriers of campylobacters, mostly C. jejuni (18). Chicken products were found to be important sources of Campylobacter infection in humans in both Asia (25) and Africa (24), and chickens were also found to be potential sources of Campylobacter bacteria in farm workers (24). Although a higher prevalence of Campylobacter on poultry products has been reported in developing countries, local customs like eating well-cooked poultry are believed to reduce the risk of infection, compared to developed countries in Europe, where the consumption of undercooked poultry is more common (18). The sources of Campylobacter infections in chickens were more likely to be horizontal contamination from the environment or the sewerage system, rather than from direct flock to flock transmission (18). The present study also showed that there is a high prevalence of Campylobacter bacteria in chickens in urban settings that have extensive indoor systems $(90.7 \%)$ than the rural settings where animals are reared under free-range conditions $(44.7 \%)(p<0.05)$. Other studies showed that the prevalence of Campylobacter colonization was higher in organic farms $(100 \%)$ compared to extensive indoor systems (49.2\%) or conventional farms (36.7\%) (26). The highest levels of recovery of Campylobacter bacteria in chickens usually occurred during the warmer months of the year (June-October) in developed countries, and more C. jejuni (43\%-86\%) than C. coli $(11 \%-39 \%)$ were recovered (18). The seasonality of Campylobacter bacteria found in poultry products in the markets coincides with the peak incidence of Campylobacter in humans, which demonstrates the importance of chicken as a source of campylobacter infection for humans in developed countries (18).

Table 1: The prevalence of thermophilic campylobacters from feces/rectal swabs of $\mathbf{4 8 5}$ different food animals in Jimma, southwest, Ethiopia

\begin{tabular}{|c|c|c|c|c|c|c|c|c|}
\hline \multirow{3}{*}{$\begin{array}{l}\text { Food } \\
\text { animals }\end{array}$} & \multicolumn{8}{|c|}{ No. of samples examined and no. of cases positive for campylobacter (\%) } \\
\hline & \multicolumn{4}{|c|}{ Urban setting } & \multicolumn{4}{|c|}{ Rural setting } \\
\hline & \multicolumn{2}{|c|}{$\mathrm{JUCA}^{\mathrm{a}}$} & \multicolumn{2}{|c|}{$\mathrm{JDDE}^{\mathrm{b}}$} & \multicolumn{2}{|c|}{ Merrewa } & \multicolumn{2}{|r|}{ Total } \\
\hline Cattle & 42 & $10(23.8)$ & 51 & $11(21.6)$ & 112 & $5(4.5)$ & 205 & $26(12.7)$ \\
\hline Chicken & 97 & $88(90.7)$ & - & - & 94 & $42(44.7)$ & 191 & $130(68.1)$ \\
\hline Pigs & 18 & $9(50.0)$ & - & - & - & - & 81 & $9(50.0)$ \\
\hline Sheep & - & - & - & - & 71 & $27(38.0)$ & 71 & $27(38.0)$ \\
\hline Total & 157 & $107(68.1)$ & 51 & $11(21.6)$ & 277 & $74(26.7)$ & 485 & $192(39.6)$ \\
\hline
\end{tabular}

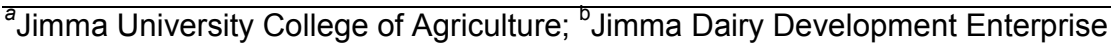

In this study, only C. coli was isolated from pigs (100\%) (Table 2). Most workers have reported a higher carriage rate of $C$. coli than $C$. jejuni among healthy pigs, providing evidence that $C$. coli is a normal flora of these animals $(10,15,16,27)$. C. jejuni is recognized as the most important causal agent of human Campylobacter enteritits, whereas C. coli is less commonly involved in human infections (28). C. coli accounted for $17.6 \%$ of the Ethiop.J.Health Dev. 2005;19(3) 
cases of human infection in Ethiopia (13). The contamination of pig carcasses during slaughter processes also represents a potential source of human infection.

Table 2: The phenotype distribution of thermophilic Campylobacter spp. from various food animals in Jimma, southwest, Ethiopia

\begin{tabular}{|c|c|c|c|}
\hline Food animals & $\begin{array}{l}\text { C. jejuni } \\
\text { No. (\%) }\end{array}$ & $\begin{array}{l}\text { C. coli } \\
\text { No. (\%) }\end{array}$ & $\begin{array}{c}\text { C. lari } \\
\text { No. (\%) }\end{array}$ \\
\hline Cattle $(n=26)$ & $14(53.8)$ & $10(38.5)$ & $2(7.7)$ \\
\hline Chickens $(n=130)$ & $105(80.8)$ & 21 (16.2) & $4(3.0)$ \\
\hline Pigs $(n=9)$ & - & $9(100.0)$ & - \\
\hline Sheep $(n=27)$ & $16(59.3)$ & $11(40.7)$ & - \\
\hline Total (192) & $135(70.3)$ & $51(26.6)$ & $6(3.1)$ \\
\hline
\end{tabular}

The frequency of Campylobacter spp. isolation (38.0\%) in sheep in this study was found to be higher than that reported in studies conducted in Norway (8.1\%) (10), Portugal (15\%) (15), and Brazil (20\%) (16). The main species of Campylobacter isolated from the faeces of sheep in this study is $C$. jejuni $(59.3 \%)$, followed by $C$. coli $(40.7 \%)$. The same pattern is observed with campylobacters isolated from the intestines of sheep at slaughter houses in Preston, Lancashire, the United Kingdom (29). Although it is likely that intestinal infection is close to $100 \%$, the shedding of campylobacters in the faeces varies considerably with the time of year (29). In some occasions, $100 \%$ of sheep were shedding camylobacters and not shedding on other occasions. This may show seasonal patterns. The shedding of campylobacters by sheep has the potential to contaminate pastures and surface waters. Contamination of surface and sub-surface waters may transmit Campylobacter within herds and between farms and other livestock groups (30).

In this study, the prevalence of thermophilic Campylobacter spp. in cattle was found to be $12.7 \%$. Previous research in cattle has been limited, but it has been increasing as outbreaks of human campylobacteriosis were traced to foods of cattle origin (18). The prevalence of Camplobacter was found to be $15 \%$ in beef from calves, $37.7 \%$ in dairy herds and $89.4 \%$ on beef cattle at slaughter (18). The prevalence in beef cattle was high in the summer, which coincides with the seasonal peak of human Campylobacter infections (18). However there are reports from different parts of the world, which show that in domestic livestock, different carriages exist ranging from 0 to $100 \%(31,32)$, with isolation most frequently seen in confined herds of dairy cattle (10). Variables, such as herd size and type, season, age of animal, sample site, sample frequency and isolation method, geography, diet and husbandry practices, have been suggested to account for the differences (33). Raw and improperly pasteurized milk from cows has been incriminated as a means of transmission of the infection in several outbreaks of milk borne campylobacteriosis $(10,34)$. Since cows might be intestinal carriers of
Campylobacter spp, the faecal contamination of milk represents a potential route leading to human infection. But on rare occasions outbreaks have been traced to asymptomatic Campylobacter mastitis in cows, which causes a high number of organisms to be directly excreted into the milk (33).

In conclusion, the results of this study indicate that thermophilic campylobacters, C. jejuni and C. coli/C. lari are very frequent among food animals in Ethiopia, suggesting possible risks of infection to people through consumption of contaminated animal products or by direct contact with infected animals. Since the available evidence shows that food animals constitute a major reservoir for these organisms, interruption of transmission to human beings from these sources should be given a high priority. Awareness of the necessity for hand washing after contact with animals and their products and the importance of proper cooking and handling of foods of animal origin are probably as important in preventing C. jejuni/coli infections as they are for preventing salmonella infections. Pasteurization of milk, chlorination of water supplies and proper cooking of foods readily kill these organisms.

\section{Acknowledgments}

This work was supported by grants made available from the School of Graduate Studies, Addis Ababa University. We thank Ato Getnet Beyene, Jimma University and Ato Gonfa Ayana, Ethiopian Health and Nutrition Institute (EHNRI), for providing some media and reagents necessary for this study. We also thank Jimma University College of Agriculture (JUCA), Jimma Dairy Development Enterprise (JDDE) and Merrewa Peasant Association for their valuable support during sample collection from farm animals.

\section{References}

1. Black RE, Levine MM, Clements ML, Hughes TP, Blaser MJ. Experimental Campylobacter jejuni infection in humans. J Infect Dis. 1988;157:472-479.

2. Robinson DA. Infective dose of Campylobacter jejuni in milk. Br Med J. 1981; 282:1584.

3. Rollins DM, Joseph SW. Campylobacter: The new leader in food-borne disease: Aetiology. Rev Med Microbiol. 2001;12:187-198

4. Blaser MJ, Duncan DJ, Warren GH, Wang WL. Experimental Campylobacter jejuni infection of adult mice. Infect Immun. 1983;39:908-916.

5. Butzler JP, Dekeyser P, Detrain M, Dehaen F. Related vibrio in stools. J Pediatr. 1973; 82:493-495.

6. Vandamme P, Falsen E, Rossau R, Hoste B, Segers P, Tytgat R, De Ley J. Revision of Campylobacter, Helicobacter, and Wolinella taxonomy: Emendation of generic descriptions and proposal of Arcobacter gen. nov. Int J Syst Bacteriol. 1991;41:88-103. 
7. On SLW: Identification methods for Campylobacters, Helicobacters, and related organisms. Clin Microbiol Rev. 1996;9:405-422.

8. Holt JG, Krieg NR, Sneath PHA, Staley JT, Williams ST: Genus Campylobacter. In: Bergey's manual of determinative bacteriology. Baltimore, MD: USA Williams \& Wilkins. 1988;41:58-61.

9. Nachamkin I, Allos BM, Ho T. Campylobacter species and Guillain-Barrè syndrome. Clin Microbiol Rev. 1998;11555-567.

10. Rosef O, Gondrosen B, Kapperud G, Underdal B. Isolation and characterization of Campylobacter jejuni and Campylobacter coli from domestic and wild mammals in Norway. Appl Environ Microbiol. 1983;46:855-859.

11. Tauxe RV. Epidemiology of Campylobacter jejuni infections in the United States and other industrialized nations. In: Campylobacter jejuni: Current Status and Future Trends. Edited by Nachamkin I, Blaser MJ, Tompkins LS. Washington, DC: American Society for Microbiology. 1992;9-19.

12. Skirrow MB, Blaser MJ: Campylobacter jejuni. In. Infections of the Gastrointestinal Tract. Edited by Blaser MJ, Smith PD, Ravdin JI, Greenberg HB, Guerrant RL. New York, Raven Press, NY USA. 1995;825-848.

13. Asrat D, Hathaway A, Ekwall E. Studies on enteric campylobacteriosis in Tikur Anbessa and EthioSwedish children's Hospital, Addis Ababa, Ethiopia. Ethiop Med J. 1999;37:71-84.

14. Gedlu E, Aseffa A. Campylobacter enteritis among children in northwest Ethiopia: A 1-year prospective study. Ann Trop Paediatr. 1996;16:207-212.

15. Cabrita J, Rodrigues J, Braganca F, Morgado C, Pires I, Goncalves AP. Prevalence, biotypes, plasmid profile and antimicrobial resistance of Campylobacter isolated from wild and domestic animals from northeast Portugal. J Appl Bacteriol. 1992;73:279-285.

16. Aquino MH, Pacheco AP, Ferreira MC, Tibana A. Frequency of isolation and identification of thermophilic campylobacters from animals in Brazil. Vet J. 2002; 164:159-161.

17. Osano $\mathrm{O}$ and Arimi S. Retail poultry and beef as source of Campylobacter jejuni. East Afri Med J. 1999;76:141-143.

18. Padungton P, Kaneene JB. Campylobacter spp. in human, chickens, pigs and their antimicrobial resistance. J Vet Med Sci; 2003;65:161-170.

19. Bolton FJ, Hutchinson DN, Coates D. Blood-free selective medium for isolation of Campylobacter jejuni from feces. J Clin Microbiol. 1984;19:169171.

20. National Committee for Clinical Laboratory Standards. 2000. Performance Standards for Antimicrobial Disk Susceptibility Tests, 7th ed. Approved standard M2-A7. National Committee for Clinical Laboratory Standards, Wayne, Pa USA.
21. Gracia M, Eaglesome M, Rigby C. Campylobacter important in veterinary medicine. Vet Bull. 1983;53: 793-818.

22. Van de Giessen AW, Bloemberg BP, Ritmeester WS, Tilburg JJ. Epidemiological study on risk factors and risk reducing measures for Campylobacter infections in Dutch broiler flocks. Epidemiol Infect. 1996;117:245-250.

23. Kazwala RR, Collins JD, Hannan J, Crinion RA, O'Mahony H. Factors responsible for the introduction and spread of Campylobacter jejuni infection in commercial poultry production. Vet Rec. 1990;126:305-306.

24. Simango C, Rukure G. Potential sources of Campylobacter species in the homes of farm workers in Zimbabwe. J Trop Med Hyg. 1991;94:388-392.

25. Rasrinaul L, Suthienkul O, Echeverria PD, Taylor DN, Seriwatana J, Bangtrakulnonth A, Lexomboon U. Foods as a source of enteropathogens causing childhood diarrhea in Thailand. Am J Trop Med Hyg. 1988;39:97-102.

26. Heuer OE, Pedersen K, Andersen JS, Madsen M. Prevalence and antimicrobial susceptibility of thermophilic Campylobacter in organic and conventional broiler flocks. Lett Appl Microbiol. 2001;33:269-274.

27. Franco DA. Campylobacter species: consideration for controlling a food-borne pathogen. J Food Prot. 1988;51:145-153.

28. Skirrow MB, Benjamin J. The classification of "thermophilic"campylobacters and their distribution in man and domestic animals, In D.G. Newell (ed.), Campylobacter. Epidemiolology, pathogenesis and biochemistry. MTP Press Limited, Lancaster, England, 1982; p. 40-44.

29. Stanley KN, Wallace JS, Currie JE, Diggle PJ, Jones K. Seasonal variation of thermophilic campylobacters in lambs at slaughter. J Appl Microbiol. 1998; 84:1111-1116.

30. Jones $\mathrm{K}$, Howard $\mathrm{S}$, Wallace JS. Intermittent shedding of thermophilic campylobacters by sheep at pasture. J Appl Microbiol. 1999;86: 531-536.

31. Firehammer BD, Myers LL. Campylobacter fetus subsp jejuni: Its possible significance in enteric disease of calves and lambs. Am J Vet Res. 1981;42:918-922.

32. Stern J. Recovery rate of Campylobacrer fetus subsp. jejuni on eviscerated pork, lamb, and beef carcasses. J Food Sci.1981;46:1291-1293.

33. Stanley K, Jones K. Cattle and sheep farms as reservoirs of Campylobacter. J Appl Microbiol. 2003;94 Suppl:104S-113S.

34. Robinson DA, Jones DM. Milk-borne Campylobacter infection. Br Med J. 1981; 282:13741376. 
